et al. Dientamoeba fragilis, a neglected cause of diarrhea, successfully treated with secnidazole. Clin Microbiol Infect 2003; 9(2): 110-113

11. van Hellemond JJ. Superior efficacy of paromomycin for eradication of Dientamoeba fragilis in adults. Abstract 335. 22nd European Congress of Clinical Microbiology and Infectious Diseases, 31 March - 3 April 2012, London.

\section{The importance of} considering sebaceous gland carcinoma in presumed chalazia in South Asian patients?

A 51-year-old Malaysian female was referred with a left lower eyelid lesion noticed 4 years ago. She consulted her GP a year ago and was diagnosed to have a chalazion. Her GP requested funding for treatment. The primary care trust (PCT) considered this a low-priority procedure and declined funding. One year later she approached her GP again and was referred to a hospital for management of this eyelid lesion (Figure 1). She underwent a biopsy and the histology was suspicious of a squamous cell carcinoma. She was referred to our unit. On examination, she had a left lower eyelid, firm $4 \mathrm{~mm}$ nodule with thickening and distortion of tarsal conjunctiva. With a clinical suspicion of sebaceous gland carcinoma (SGC), a wedge excision of the lesion was performed. Paraffin section histology confirmed complete excision of SGC. Delayed repair required a Tenzel flap. She remains asymptomatic at 5-month follow-up.

\section{Discussion}

Eyelid SGC is known to masquerade as chalazion. ${ }^{1}$ Patients with longer duration of symptoms have been reported to have more advanced disease and higher rates of recurrence or metastasis. Delay in diagnosis and treatment of SGC by more than 6 months has been reported to be associated with increased incidence of recurrence. ${ }^{2}$ In this case of SGC the treatment was denied by a PCT, after mis-diagnosis as a chalazion, considering it as a low priority procedure.

Although criteria may vary, PCTs only fund excision of chalazion when the following criteria are met: the chalazion has been present for more than 6 months, it is situated on the upper eyelid, and it is causing blurring of vision.

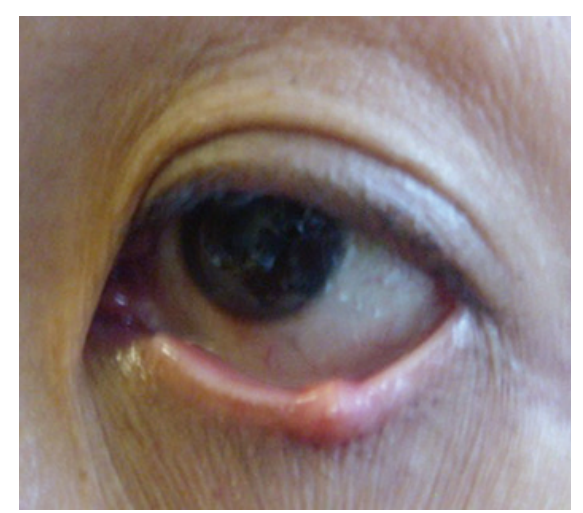

Figure 1. Lower eyelid lesion.

SGC is more common in the upper eyelid than lower, with ratios varying from 1.3 to 3. The above PCT criteria could lead to a delay in diagnosis of a SGC masquerading as chalazion in the lower eyelids. SGC is rare in the white population, accounting for $1-5.5 \%$ of all eyelid malignancies.,4 However, SGC appears to be much more common in the Asian population (South Asian and Indian), accounting for 27-40\% of all eyelid malignancies. ${ }^{3.4}$ Hence, the possibility of SGC should be considered in Asian individuals presenting with persistent chalazion. Persistent or recurrent chalazion and increase in size of lesion particularly in individuals of Asian origin should justify early referral to the ophthalmologist.

\section{Suresh Sagili,}

FRCOphth, Corneo Plastic Unit, Queen Victoria Hospital, East Grinstead, West Sussex, RH19 3DZ.

Email: sureshsagilidyahoo.com

Raman Malhotra,

FRCOphth, Corneo Plastic Unit, Queen Victoria Hospital, East Grinstead.

\section{REFERENCES}

1. Ozdal PC, Codère F, Callejo $S$, et al. Accuracy of the clinical diagnosis of chalazion. Eye 2004 18(2): 135-138.

2. Song A, Carter KD, Syed NA, et al. Sebaceous cell carcinoma of the ocular adnexa: clinical presentations, histopathology, and outcomes. Ophthal Plast Reconstr Surg 2008; 24(3): $194-$ 200

3. Takamura H, Yamashita H. Clinicopathological analysis of malignant eyelid tumor cases at Yamagata university hospital: statistical comparison of tumor incidence in Japan and in other countries. Jpn J Opthalmol 2005; 49(5): 349-354.

4. Wang JK, Liao SL, Jou JR, et al. Malignant eyelid tumours in Taiwan. Eye (Lond) 2003; 17(2): 216-220

DOI: 10.3399/bjgp13X663037 